\title{
Providing Performance Guarantees for Buffered Crossbar Switches without Speedup
}

\author{
Deng Pan, Zhenyu Yang, Kia Makki, and Niki Pissinou \\ Florida International University \\ Miami, FL 33199 \\ \{pand, yangz, makkik, pissinou\}@fiu.edu
}

\begin{abstract}
Buffered crossbar switches are special crossbar switches with each crosspoint equipped with a small exclusive buffer. The crosspoint buffers decouple input ports and output ports, and simplify switch scheduling. In this paper, we propose a scheduling algorithm called Fair and Localized Asynchronous Packet Scheduling (FLAPS) for buffered crossbar switches, to provide tight performance guarantees. FLAPS needs no speedup for the crossbar and handles variable length packets without segmentation and reassembly (SAR). With FLAPS, each input port and output port independently make scheduling decisions and rely on only local queue statuses. We theoretically show that a crosspoint buffer size of $4 L$ is sufficient for FLAPS to avoid buffer overflow, where $L$ is the maximum packet length. In addition, we prove that FLAPS achieves strong stability, and provides bounded delay guarantees. Finally, we present simulation data to verify the analytical results.
\end{abstract}

Key words: Buffered crossbar switches, performance guarantees, speedup, stability.

\section{Introduction}

Crossbar switches provide nonblocking capabilities, and overcome the bandwidth limitation of bus based switches [1]. They have long been used as high speed interconnects in various computing environments, such as Internet routers, computer clusters, and system-on-chip networks. Traditional crossbar switches have no buffers at the crosspoints of the crossbar switching fabric, and packets have to be directly transmitted from input ports to output ports. Such switches work in a synchronous time slot mode and handle only fixed length cells [2]. When variable length packets arrive, they need to be segmented into fixed length cells at input ports. The cells are then used as the scheduling units and transmitted to output ports, where they are reassembled into original packets and sent to the output lines. This process is called segmentation and reassembly (SAR) [3].

With the development of VLSI technology, it has been feasible to integrate on-chip memories to the crossbar [4] - [7]. Buffered crossbar switches are a special type of crossbar switches, which have a small exclusive buffer at each crosspoint, as shown in Figure 1. Crosspoint buffers decouple input ports and output ports, and greatly simplify the scheduling process. Buffered crossbar switches can now directly handle variable length packets and work in an asynchronous mode. To 
be specific, input ports independently and periodically send packets of arbitrary length to their crosspoint buffers, from where output ports retrieve the packets one by one.

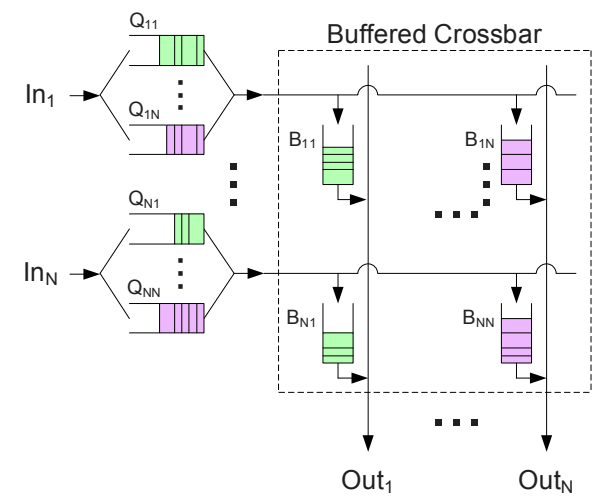

Fig. 1. Structure of buffered crossbar switches.

Compared with (fixed length) cell scheduling of unbuffered crossbar switches, (variable length) packet scheduling of buffered crossbar switches has some unique advantages. First, packet scheduling can better utilize available bandwidth and achieve higher throughput. For cell scheduling, when a packet is segmented into cells, its length may not be a multiple of the cell length, and padding bits have to be inserted to the last segment to reach the cell length. The padding bits do not contain useful information and waste bandwidth. In the worst case, if all packets have a slightly longer length than the cell length, each packet has to be segmented into two cells, and the switch can only achieve about a half of its maximum capacity [14]. Second, packet scheduling reduces packet latency, and helps achieve tight performance guarantees. Because there is no SAR, packets arriving at input ports can be immediately transmitted, and packets received at output ports can be immediately sent to the output lines. Third, no extra buffer space is necessary at input ports and output ports for SAR, which lowers hardware cost. In cell scheduling, an input port of an $N \times N$ switch may alternatively send segments of $N$ packets to the $N$ different output ports, and similarly an output port may receive segments from $N$ input ports. Thus, $N L$ buffer space is needed at each input port and output port for SAR, where $L$ is the maximum packet length. Finally, cell scheduling is a special case of packet scheduling, or in other words, packet scheduling can also handle fixed length cells.

The speedup of a switch defines the ratio of the crossbar speed to the input or output port speed. To be specific, speedup of $S$ means that the crossbar has $S$ times bandwidth as that of the input port or output port. Obviously, with the same crossbar, the larger the speedup requirement is, the smaller the switch capacity is. The buffered crossbar switches considered in this paper do not need speedup. Because the crossbar runs at the same speed as the output port, no buffer space is necessary at the output port. When a packet is transmitted to the output port, it will be immediately sent to the next hop via the output line. 
There are a number of scheduling algorithms for buffered crossbar switches in the literature. Those algorithms can be classified into two categories: to provide performance guarantees [8] - [14] and to achieve high throughput [15] - [20]. The former requirement is stronger than the latter. In other words, an algorithm with tight performance guarantees usually delivers $100 \%$ throughput, but the reverse is not always true. Among the scheduling algorithms providing performance guarantees, some consider cell scheduling [8] - [13]. As discussed in the above, cell scheduling may waste bandwidth due to the padding bits in SAR. Others require speedup of two or more [9] - [14], which reduces the maximum capacity of the switch by half. In particular, two packet scheduling algorithms, Packet GVOQ (PGV) and Packet LOOFA (PLF), were proposed for buffered crossbar switches in [14], and their performance guarantees were analyzed. There are two main differences between the algorithms in [14] and our algorithm. First, PGV and PLF work by emulating push-in-first-out (PIFO) scheduling algorithms for output queued $(\mathrm{OQ})$ switches, which means that they need to maintain the operation of the reference algorithms. Second, PGV and PLF require speedup of two for the crossbar. To the best of our knowledge, there have been no existing packet scheduling algorithms for buffered crossbar switches without speedup.

In this paper, we propose the Fair and Localized Asynchronous Packet Scheduling (FLAPS) algorithm for buffered crossbar switches without speedup. FLAPS allows input ports and output ports to make independent scheduling decisions based on only local information without data exchange. More specifically, an input port needs only the statuses of its input queues, and an output port needs only the states of its crosspoint buffers. FLAPS uses a time stamp based approach to schedule packets. We show that FLAPS has a crosspoint buffer size bound of $4 L$, independent of the switch size. Furthermore, we prove that FLAPS achieves strong stability, and provides bounded performance guarantees. Finally, simulations are conducted to verify the analytical results and evaluate the performance of FLAPS.

The rest of the paper is organized as follows. In Section 2, we discuss the ideal fairness model that will be used. In Section 3, we present the FLAPS algorithm. In Section 4, we theoretically analyze the performance of FLAPS, and in Section 5 , we present simulation data to verify the analytical results. In Section 6 , we conclude the paper.

\section{Preliminaries}

In this section, we discuss the ideal fairness model that will be used in this paper. To effectively evaluate the fairness performance of a scheduling algorithm, it is necessary to have an ideal fairness model as the comparison reference. A fairness model for packet scheduling can be regarded to have two roles. The first role is to calculate the allocated bandwidth for traffic flows based on their requested bandwidth. The second role is to schedule the packets of different flows to ensure that the actually received bandwidth of each flow is equal to its allocated bandwidth.

Generalized Processor Sharing (GPS) [21] is a widely used fairness model for packet scheduling. When GPS applies to a shared output link, it divides 
the link bandwidth into multiple logical transmission channels. Each flow has its own logical channel, and the channel bandwidth is proportional to the requested bandwidth of the flow. GPS views flows as fluids of continuous bits, and transmits the packets of a flow in its independent channel. As a result, each flow uses the same amount of bandwidth as that of its allocated bandwidth. To improve utilization, when a flow temporarily becomes empty, GPS will reallocate the leftover bandwidth of the empty flow to the remaining backlogged flows in proportion to their requested bandwidth.

As can be seen, when GPS serves a shared output link, it allocates available bandwidth, including leftover bandwidth, in the proportional manner. However, simple proportional bandwidth allocation is not proper for switch scheduling [22] [23]. The reason is that, while flows of a shared output link are constrained only by the link bandwidth, flows of a switch are subject to two bandwidth constraints: the available bandwidth at both the input port and output port of the flow. Naive bandwidth allocation at the output port may make the flows violate the bandwidth constraints at their input ports, and vice versa.

Fair bandwidth allocation for switches is an interesting problem, and there are algorithms [22] [23] in the literature to solve it. In this paper, we assume that bandwidth allocation has been calculated using such algorithms, and the scheduling algorithms just schedule packets to ensure the allocated bandwidth of each flow. Also, when a flow of the switch temporarily becomes empty, we do not assume that its allocated bandwidth is immediately reallocated. Instead, the bandwidth allocation algorithms will consider the leftover bandwidth in the next calculation. Bandwidth allocation is recalculated when requested bandwidth changes or existing backlogged flows become empty. Given the calculated bandwidth allocation, GPS can divide the bandwidth of a switch into logical channels, as shown in Figure 2. Each flow has an independent logical channel, and the channel bandwidth is equal to the allocated bandwidth of the flow. Thus, traffic of the flow can smoothly stream from the input port to the output port.

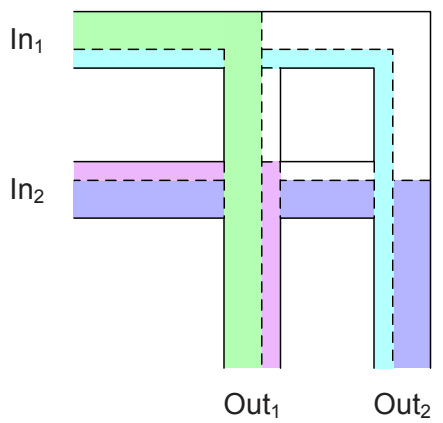

Fig. 2. GPS used for switch scheduling.

To sum up, we use GPS as the ideal fairness model only for its second role, i.e., given the allocated bandwidth, to compare the received bandwidth of a flow in our algorithm and in GPS. 


\section{Fair and Localized Asynchronous Packet Scheduling}

In this section, we present the Fair and Localized Asynchronous Packet Scheduling (FLAPS) algorithm.

The switch structure that we consider is shown in Figure 1. $N$ input ports and $N$ output ports are connected by a buffered crossbar, which has no speedup. Denote the $i^{t h}$ input port as $I n_{i}$ and the $j^{t h}$ output port as $O u t_{j}$. Use $R$ to represent the available bandwidth of each input port and output port, and the crossbar also has bandwidth $R$. Each input port has a buffer organized as virtual output queues (VOQ) [24], i.e., there are $N$ virtual queues to store the packets destined to the $N$ different output ports. Denote the virtual queue at $I n_{i}$ for packets to $O u t_{j}$ as $Q_{i j}$. Each crosspoint has a small exclusive buffer. Denote the crosspoint buffer connecting $I n_{i}$ and $O u t_{j}$ as $B_{i j}$. Output ports have no buffers. After a packet arrives at the switch, it is first stored in the input queue, and waits to be sent to the crosspoint buffer. The packet will then be sent from the crosspoint buffer to the output port and immediately delivered to the output line. We say a packet arrives at or departs from a buffer when its last bit arrives at or departs from the buffer.

Define the traffic from $I n_{i}$ to $O u t_{j}$ to be a flow $F_{i j}$. Because we consider performance guarantees in this paper, each flow has explicit allocated bandwidth, and the objective of the scheduling algorithm is to ensure that each flow receives the same amount of bandwidth as that of its allocated bandwidth. Use $r_{i j}(t)$ to represent the allocated bandwidth of $F_{i j}$, which is a function of time $t$ with discrete values in practice. Bandwidth allocation is calculated by the algorithms mentioned in Section 2. The calculated bandwidth allocation should be feasible, i.e., no over-subscription at any input port or output port

$$
\forall i, \sum_{x=1}^{N} r_{i x}(t) \leq R, \text { and } \forall j, \sum_{x=1}^{N} r_{x j}(t) \leq R
$$

The feasibility requirement is only for bandwidth allocation. It is necessary because it is impossible to allocate more bandwidth than what is actually available. However, it does not mean that no temporary overload is allowed for an input port or output port. As will be seen in Section 4.2, we use the extended leaky bucket scheme for flow admission control. It allows any flow to be overloaded for an arbitrarily long period with an arbitrarily large but finite burst.

There are two types of scheduling in the switch, which we call input scheduling and output scheduling. In input scheduling, an input port selects a packet from one of its input queues, and sends it to the corresponding crosspoint buffer. In output scheduling, an output port selects a packet from one of its crosspoint buffers, and sends it to the output line. When we need to differentiate the scheduling algorithms used for input scheduling and output scheduling, we use the notation "A-B". A is the scheduler for input scheduling, and B is the scheduler for output scheduling. A and B could be either FLAPS or GPS. If we do not care the scheduler for output scheduling, we use a $*$ mark for B. For example, GPS-GPS means that GPS is used for both input scheduling and output scheduling. In such a scenario, each flow has an independent channel, and 
its traffic moves smoothly from the input output to the output port without buffering in the middle, as shown in Figure 2.

Input scheduling and output scheduling of FLAPS rely on only local information, and are conducted in an asynchronous and distributed manner. To be specific, an input port needs only the statuses of the queues in its input buffer, and does not exchange information with any crosspoint buffer or output port. Similarly, an output port needs only the statuses of its crosspoint buffers.

We first describe the input scheduling of FLAPS, which uses time stamps as scheduling criteria. There are two types of time stamps. For easy representation, we denote the $k^{t h}$ arrived packet of $F_{i j}$ as $P_{i j}^{k}$. The first time stamp for $P_{i j}^{k}$ is called virtual input start time, denoted as $\widehat{I S}_{i j}^{k}$, which is the service start time of $P_{i j}^{k}$ at the input port in GPS-*. The second time stamp is virtual input finish time, denoted as $\widehat{I F}_{i j}^{k}$, which is the service finish time of $P_{i j}^{k}$ at the input port in GPS-*. In other words, if GPS is the scheduler for input scheduling, $\widehat{I S}_{i j}^{k}$ and $\widehat{I F}_{i j}^{k}$ are the time that the first bit and last bit of $P_{i j}^{k}$ leave $Q_{i j}$, respectively. $\widehat{I S}_{i j}^{k}$ can be calculated as follows

$$
\widehat{I S}_{i j}^{k}=\max \left(I A_{i j}^{k}, \widehat{I F}_{i j}^{k-1}\right)
$$

where $I A_{i j}^{k}$ is the arrival time of $P_{i j}^{k}$ at the input port. $\widehat{I F}_{i j}^{k}$ satisfies the following relationship

$$
\int_{\widehat{I S}_{i j}^{k}}^{\widehat{I F}_{i j}^{k}} r_{i j}(x) d x=L_{i j}^{k}
$$

where $L_{i j}^{k}$ is the length of $P_{i j}^{k}$. Because $r_{i j}(t)$ has only discrete values in practice, $\widehat{I F}_{i j}^{k}$ can be easily calculated. For example, if $r_{i j}(t)$ is a constant $r_{i j}$ during $\left[\widehat{I S}_{i j}^{k}, \widehat{I F}_{i j}^{k}\right], \widehat{I F}_{i j}^{k}$ can be calculated as

$$
\widehat{I F}_{i j}^{k}=\widehat{I S}_{i j}^{k}+\frac{L_{i j}^{k}}{r_{i j}}
$$

In the first step of input scheduling of FLAPS, $I n_{i}$ identifies eligible packets. A packet $P_{i j}^{k}$ is eligible for input scheduling if its virtual input start time $\widehat{I S}_{i j}^{k}$ is smaller than or equal to the current system time $t$. In other words, a packet that has started transmission in GPS-* is eligible in FLAPS-*. If there exist eligible packets in the input buffer, $I n_{i}$ will select among such packets the one $P_{i j}^{k}$ with the smallest virtual input finish time $\widehat{I F}_{i j}^{k}$, and send it to $B_{i j}$. If there are no eligible packets, $I n_{i}$ will wait until the next earliest virtual input start time of a packet. Note that when $I n_{i}$ is waiting for an eligible packet, if an empty input queue has a new incoming packet, whose virtual input start time is equal to its arrival time, $I n_{i}$ should immediately start transmitting this new packet. For easy reading, the pseudo code description for input scheduling of FLAPS is given in Table 1. 
Table 1. Input Scheduling of FLAPS

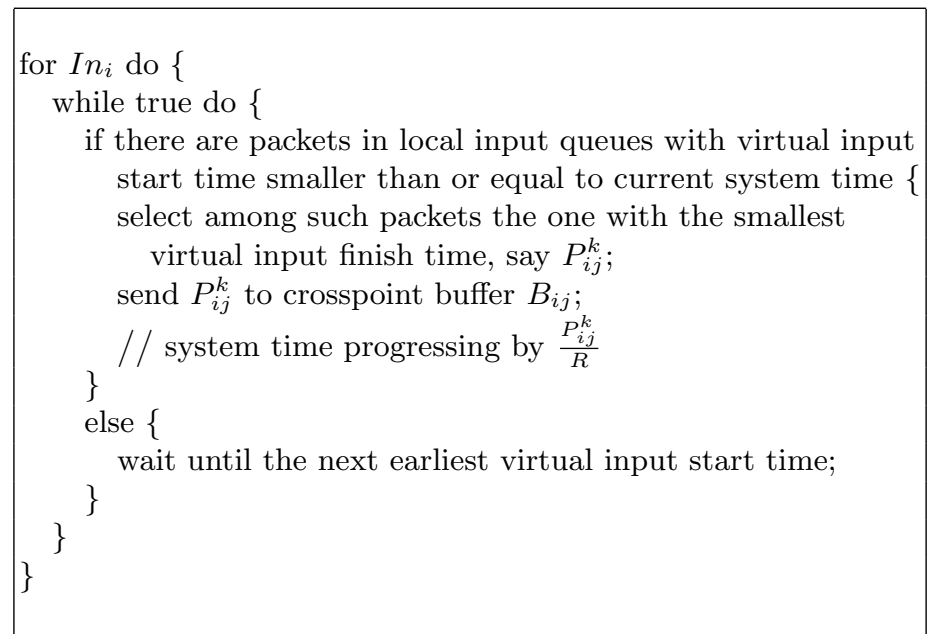

We denote the actual input start time and finish time of $P_{i j}^{k}$ in FLAPS-* as $I S_{i j}^{k}$ and $I F_{i j}^{k}$, which are the time that the first bit and the last bit of $P_{i j}^{k}$ leave $Q_{i j}$ in FLAPS-*, respectively. Apparently

$$
I F_{i j}^{k}=I S_{i j}^{k}+\frac{L_{i j}^{k}}{R}
$$

Output scheduling of FLAPS is similar to input scheduling. There are also several time stamps for $P_{i j}^{k}$. The virtual output start time $\widehat{O S}_{i j}^{k}$ and virtual output finish time $\widehat{O F}_{i j}^{k}$ are the time that the first bit and last bit of $P_{i j}^{k}$ leave $B_{i j}$ in FLAPS-GPS, respectively. In other words, after $P_{i j}^{k}$ is delivered to $B_{i j}$ by FLAPS, if GPS is the scheduler for output scheduling, $P_{i j}^{k}$ will start transmission at $\widehat{O S}_{i j}^{k}$ and finish at $\widehat{O F}_{i j}^{k} . \widehat{O S}_{i j}^{k}$ is calculated as

$$
\widehat{O S}_{i j}^{k}=\max \left(O A_{i j}^{k}, \widehat{O F}_{i j}^{k-1}\right)
$$

where $O A_{i j}^{k}$ is the arrival time of $P_{i j}^{k}$ at $B_{i j}$ in FLAPS- ${ }^{*}$, and is equal to $I F_{i j}^{k}$ by neglecting the propagation delay. $\widehat{O F}_{i j}^{k}$ satisfies the following relationship

$$
\int_{\widehat{O S}_{i j}^{k}}^{\widehat{O F}_{i j}^{k}} r_{i j}(x) d x=L_{i j}^{k}
$$

Similarly, in output scheduling of FLAPS, $O u t_{j}$ first identifies eligible packets, and a packet $P_{i j}^{k}$ is eligible if its virtual output start time $\widehat{O S}_{i j}^{k}$ is smaller than or equal to the current system time $t$. If there are eligible packets in the crosspoint buffers, Out ${ }_{j}$ retrieves the one $P_{i j}^{k}$ with the smallest virtual output finish time $\widehat{O F}_{i j}^{k}$, and sends it to the output line. Otherwise, it waits until there is 
Table 2. Output Scheduling of FLAPS

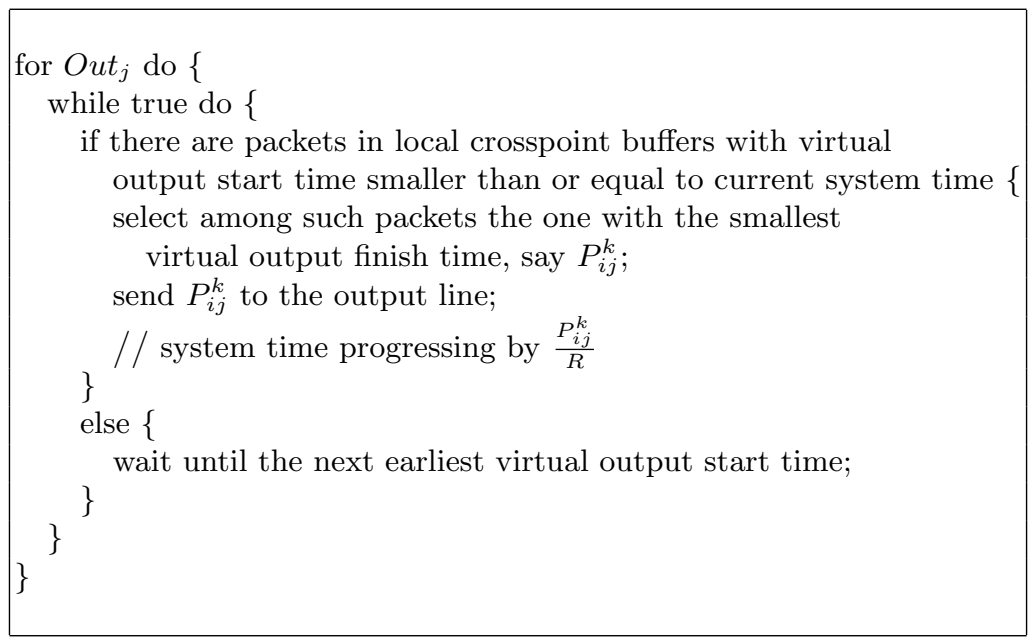

an eligible packet. The pseudo code description for output scheduling of FLAPS is given in Table 2 .

Correspondingly, $O S_{i j}^{k}$ and $O F_{i j}^{k}$ are the actual output start and finish time of $P_{i j}^{k}$, which are the time that the first bit and the last bit of $P_{i j}^{k}$ leave $B_{i j}$ in FLAPS-FLAPS, respectively. It is obvious that

$$
O F_{i j}^{k}=O S_{i j}^{k}+\frac{L_{i j}^{k}}{R}
$$

\section{Performance Analysis}

In this section, we theoretically analyze the performance of FLAPS. We show that FLAPS has a bounded crosspoint buffer size, achieves strong stability, and provides tight delay guarantees.

\subsection{Crosspoint Buffer Size Bound}

To avoid overflow at crosspoint buffers, we would like to find the maximum number of bits buffered at any crosspoint.

Based on the description of the FLAPS algorithm, we have the following properties.

Property 1 For any packet, its actual input start time in FLAPS- * is larger than or equal to its virtual input start time in GPS-*, i.e.,

$$
I S_{i j}^{k} \geq \widehat{I S}_{i j}^{k}
$$

Property 2 For any packet, its actual output start time in FLAPS-FLAPS is larger than or equal to its virtual output start time in FLAPS-GPS, i.e.,

$$
O S_{i j}^{k} \geq \widehat{O S}_{i j}^{k}
$$


First, we define some notations for input scheduling. We say that $Q_{i j}$ is backlogged at time $t$, if there exists $k$ such that $\widehat{I S}_{i j}^{k} \leq t \leq \widehat{I F}_{i j}^{k}$. Intuitively, $Q_{i j}$ is backlogged at $t$ if $Q_{i j}$ has buffered bits at $t$ in GPS-*. Define $\hat{q}_{i j}(t)$ to represent the backlog status of $Q_{i j}$ at $t . \hat{q}_{i j}(t)=1$ or 0 means that $Q_{i j}$ is backlogged or empty at $t$.

Use $t o B_{i j}\left(t_{1}, t_{2}\right)$ and $\widehat{t o B}_{i j}\left(t_{1}, t_{2}\right)$ to represent the numbers of bits transmitted by $F_{i j}$ from $I n_{i}$ to $B_{i j}$ during interval $\left[t_{1}, t_{2}\right]$ in FLAPS-* and GPS-*, respectively. Based on the definition of GPS, $\widehat{t o B}_{i j}\left(t_{1}, t_{2}\right)$ can be calculated as

$$
\widehat{t o B}_{i j}\left(t_{1}, t_{2}\right)=\int_{t_{1}}^{t_{2}} r_{i j}(x) \hat{q}_{i j}(x) d x
$$

Next, we define some corresponding notations for output scheduling. We say that $B_{i j}$ is backlogged at time $t$, if there exists $k$ such that $\widehat{O S}_{i j}^{k} \leq t \leq \widehat{O F}_{i j}^{k}$. Define $\hat{b}_{i j}(t)$ to represent the backlog status of $B_{i j}$ at $t . \hat{b}_{i j}(t)=1$ or 0 means that $B_{i j}$ is backlogged or empty at $t$.

Use $t_{o} O_{i j}\left(t_{1}, t_{2}\right)$ and $\widehat{t o O}_{i j}\left(t_{1}, t_{2}\right)$ to represent the numbers of bits transmitted by $F_{i j}$ from $B_{i j}$ to $O u t_{j}$ during interval $\left[t_{1}, t_{2}\right]$ in FLAPS-FLAPS and FLAPS-GPS, respectively. $\widehat{t o O}_{i j}\left(t_{1}, t_{2}\right)$ can be calculated as

$$
\widehat{t o O}_{i j}\left(t_{1}, t_{2}\right)=\int_{t_{1}}^{t_{2}} r_{i j}(x) \hat{b}_{i j}(x) d x
$$

The following lemma gives the relationship between the service time of a packet in FLAPS-* and GPS-*.

Lemma 1 For any packet, its actual input start time in FLAPS-* is less than or equal to its virtual input finish time in GPS-*, i.e.,

$$
I S_{i j}^{k} \leq \widehat{I F}_{i j}^{k}
$$

The proofs of Lemmas 1 to 4 are similar to the performance analysis of $\mathrm{WF}^{2} \mathrm{Q}$ in [25]. They are omitted in this paper due to space limitations.

Correspondingly, there is a lemma for output scheduling.

Lemma 2 For any packet, its actual output start time in FLAPS-FLAPS is less than or equal to its virtual output finish time in FLAPS-GPS, i.e.,

$$
O S_{i j}^{k} \leq \widehat{O F}_{i j}^{k}
$$

The next lemma compares $t o B_{i j}\left(t_{1}, t_{2}\right)$ and $\widehat{t o B}_{i j}\left(t_{1}, t_{2}\right)$.

Lemma 3 During interval $[0, t]$, the difference between the number of bits sent from input port In $n_{i}$ to crosspoint buffer $B_{i j}$ in FLAPS-* and GPS-* is greater than or equal to $-L$ and less than or equal to $L$, i.e.,

$$
-L \leq t o B_{i j}(0, t)-\widehat{t o B}_{i j}(0, t) \leq L
$$

For output scheduling, there is a similar lemma as follows. 
Lemma 4 During interval $[0, t]$, the difference between the number of bits sent from crosspoint buffer $B_{i j}$ to output port Out ${ }_{j}$ in FLAPS-FLAPS and FLAPSGPS is greater than or equal to $-L$ and less than or equal to $L$, i.e.,

$$
-L \leq t o O_{i j}(0, t)-t_{o O} O_{i j}(0, t) \leq L
$$

The next lemma compares the number of bits transmitted by the same flow in the input scheduling of GPS-* and the output scheduling of FLAPS-GPS.

Lemma 5 During interval $[0, t]$, the number of bits transmitted by flow $F_{i j}$ from input port I $n_{i}$ to crosspoint buffer $B_{i j}$ in GPS- * is less than or equal to that from crosspoint buffer $B_{i j}$ to output port Out ${ }_{j}$ in FLAPS-GPS plus $2 L$, i.e.,

$$
\widehat{t o B}_{i j}(0, t) \leq \widehat{t o O}_{i j}(0, t)+2 L
$$

Proof. Assume that $B_{i j}$ in FLAPS-GPS is empty immediately before time $s$ and is continuously backlogged during $[s, t]$. If $B_{i j}$ is not backlogged at $t$, then $s=t$.

By Lemma 3, we have $t_{o} B_{i j}(0, s) \geq \widehat{t o B}_{i j}(0, s)-L$. Because $B_{i j}$ is empty before $s$ and backlogged after $s$ in FLAPS-GPS, all packets arriving at $B_{i j}$ before $s$ have been transmitted to $O u t_{j}$, and a new packet arrives at $B_{i j}$ at $s$. Thus

$$
\begin{aligned}
\widehat{t o O}_{i j}(0, s) & \geq t_{o B_{i j}}(0, s)-L \\
& \geq \widehat{t o B}_{i j}(0, s)-2 L
\end{aligned}
$$

On the other hand, because $B_{i j}$ is continuously backlogged during $[s, t], \hat{b}_{i j}(t)$ is equal to 1 in the interval. Therefore

$$
\begin{aligned}
\widehat{t o O}_{i j}(s, t) & =\int_{s}^{t} r_{i j}(x) \hat{b}_{i j}(x) d x \\
& =\int_{s}^{t} r_{i j}(x) d x \\
& \geq \int_{s}^{t} r_{i j}(x) \hat{q}_{i j}(x) d x \\
& =\widehat{t o B}_{i j}(s, t)
\end{aligned}
$$

Adding (18) and (19), we obtain

$$
\widehat{t o O}_{i j}(0, t) \geq \widehat{t o B}_{i j}(0, t)-2 L
$$

The following theorem gives the bound for the crosspoint buffer size.

Theorem 1 In FLAPS-FLAPS, the maximum number of bits buffered at a crosspoint buffer is upper bounded by $4 L$, i.e.,

$$
t_{o} B_{i j}(0, t)-t_{o} O_{i j}(0, t) \leq 4 L
$$

Proof. By Lemma 4,

$$
t o O_{i j}(0, t)+L \geq \widehat{t o O}_{i j}(0, t)
$$

By Lemma 5,

$$
\widehat{t o O}_{i j}(0, t)+2 L \geq \widehat{t o B}_{i j}(0, t)
$$

By Lemma 3,

$$
\widehat{t o B}_{i j}(0, t)+L \geq t o B_{i j}(0, t)
$$

Summing the above equations, we have proved the theorem. 


\subsection{Switch Stability}

We have shown in the above that FLAPS has a bounded crosspoint buffer size. In this subsection, we show that the lengths of input virtual queues are finite, and thus FLAPS achieves strong stability.

As discussed in Section 3, because each flow is allocated a specific amount of bandwidth, it is necessary to have admission control for the flow to avoid over-subscription. The leaky bucket scheme [1] is a widely used traffic shaping scheme, and we will use it for admission control. In the classical definition of a leaky bucket, the flow rate is a constant, which we extend in this paper to be a variable. The reason is that the allocated bandwidth of a flow may change after bandwidth allocation calculations. Use $t o I_{i j}\left(t_{1}, t_{2}\right)$ to denote the number of incoming bits of $F_{i j}$ during interval $\left[t_{1}, t_{2}\right]$. If $F_{i j}$ is leaky bucket $\left(r_{i j}(t), \sigma_{i j}\right)$ complaint, then during any interval $\left[t_{1}, t_{2}\right]$

$$
t o I_{i j}\left(t_{1}, t_{2}\right) \leq \int_{t_{1}}^{t_{2}} r_{i j}(x) d x+\sigma_{i j}
$$

where $\sigma_{i j}$ can be an arbitrary positive constant and is called the burst size of $F_{i j}$. Intuitively, during any time interval, $F_{i j}$ can have $\sigma_{i j}$ more incoming traffic than what it can transmit.

Use $Q_{i j}(t)$ to represent the queue occupancy of $Q_{i j}$ at $t$, i.e. the number of bits buffered in $Q_{i j}$ at $t$. Define $X(t)=\left(Q_{11}(t), \ldots, Q_{i j}(t), \ldots, Q_{N N}(t)\right)$, and use $\|X\|$ to represent the Euclidean norm of vector $X=\left(x_{1}, x_{2}, \ldots, x_{n}\right)$, i.e.

$$
\|X\|=\sqrt{\sum_{i=1}^{n} x_{i}^{2}}
$$

Following the definition in [26], we say that a system of queues is strongly stable if

$$
\lim _{n \rightarrow \infty} \sup E[\|X(t)\|]<\infty
$$

Note that strong stability implies $100 \%$ throughput [26].

Theorem 2 When flows are leaky bucket complaint, FLAPS is strongly stable.

Proof. Assume that flow $F_{i j}$ is leaky bucket $\left(r_{i j}(t), \sigma_{i j}\right)$ compliant. Also assume that $Q_{i j}$ is empty immediately before $s$ and continuously backlogged during $[s, t]$. This indicates that all packets of $F_{i j}$ arriving at $Q_{i j}$ before $s$ have finished transmission by $s$ in GPS-*, and the next packet has not arrived. Therefore

$$
t_{o I}(0, s) \leq \widehat{\operatorname{toB}}_{i j}(0, s)+L
$$

During $[s, t], Q_{i j}$ is continuously backlogged, and thus

$$
\widehat{t o B}_{i j}(s, t)=\int_{s}^{t} r_{i j}(x) \hat{q}_{i j}(x) d x=\int_{s}^{t} r_{i j}(x) d x
$$

Because the arrival traffic is leaky bucket $\left(r_{i j}(t), \sigma_{i j}\right)$ compliant, we have

$$
{ }_{t o I} I_{i j}(s, t) \leq \int_{s}^{t} r_{i j}(x) d x+\sigma_{i j}
$$


By (28), (29), and (30)

$$
t o I_{i j}(0, t) \leq \widehat{t o B}_{i j}(0, t)+L+\sigma_{i j}
$$

We know from Lemma 3 that $\widehat{t o B}_{i j}(0, t) \leq t o B_{i j}(0, t)+L$. Thus

$$
\begin{aligned}
t_{o I} I_{i j}(0, t) & \leq \widehat{t o B}_{i j}(0, t)+L+\sigma_{i j} \\
& \leq t o B_{i j}(0, t)+2 L+\sigma_{i j}
\end{aligned}
$$

By the definition of $Q_{i j}(t)$, we can obtain

$$
Q_{i j}(t)=t o I_{i j}(0, t)-t o B_{i j}(0, t) \leq 2 L+\sigma_{i j}
$$

Since both $L$ and $\sigma_{i j}$ are finite, we have

$$
\|X(t)\|=\sqrt{\sum_{i=1}^{N} \sum_{j=1}^{N} Q_{i j}(t)^{2}}<\infty
$$

\subsection{Delay Guarantees}

In this subsection, we show that FLAPS can provide bounded delay guarantees. For easy analysis, we assume that the allocated bandwidth $r_{i j}(t)$ of $F_{i j}$ is a constant $r_{i j}$ during interval $\left[\min \left(I S_{i j}^{k}, \widehat{I S}_{i j}^{k}\right), \max \left(O F_{i j}^{k}, \widehat{O F}_{i j}^{k}\right)\right]$.

Use $\widetilde{O F}_{i j}^{k}$ to denote the departure time of $P_{i j}^{k}$ in GPS-GPS. By neglecting the propagation delay, we have $\widetilde{O F}_{i j}^{k}=\widehat{I F}_{i j}^{k}$. Similarly, $O F_{i j}^{k}$ is the departure time of packet $P_{i j}^{k}$ in FLAPS-FLAPS, if the propagation delay is neglected.

Theorem 3 The difference between the departure time of packet $P_{i j}^{k}$ in FLAPSFLAPS and GPS-GPS is greater than or equal to $-L_{i j}^{k}\left(\frac{1}{r_{i j}}-\frac{2}{R}\right)$ and less than or equal to $L\left(\frac{3}{r_{i j}}+\frac{2}{R}\right)$, i.e.

$$
-L_{i j}^{k}\left(\frac{1}{r_{i j}}-\frac{2}{R}\right) \leq O F_{i j}^{k}-\widetilde{O F}_{i j}^{k} \leq L\left(\frac{3}{r_{i j}}+\frac{2}{R}\right)
$$

Proof. First, we prove $O F_{i j}^{k}-\widetilde{O F}_{i j}^{k} \geq-L_{i j}^{k}\left(\frac{1}{r_{i j}}-\frac{2}{R}\right)$. It is obvious that

$$
O F_{i j}^{k} \geq O A_{i j}^{k}+\frac{L_{i j}^{k}}{R}=I F_{i j}^{k}+\frac{L_{i j}^{k}}{R}
$$

Based on Property 1, we know $\widehat{I S}_{i j}^{k} \leq I S_{i j}^{k}$ or in other words $\widehat{I F}_{i j}^{k}-\frac{L_{i j}^{k}}{r_{i j}} \leq$ $I F_{i j}^{k}-\frac{L_{i j}^{k}}{R}$, and thus we obtain

$$
\begin{aligned}
\widetilde{O F}_{i j}^{k} & =\widehat{I F}_{i j}^{k} \\
& \leq I F_{i j}^{k}+L_{i j}^{k}\left(\frac{1}{r_{i j}}-\frac{1}{R}\right) \\
& \leq O F_{i j}^{k}+L_{i j}^{k}\left(\frac{1}{r_{i j}}-\frac{2}{R}\right)
\end{aligned}
$$


Next, we prove $O F_{i j}^{k}-\widetilde{O F}_{i j}^{k} \leq L\left(\frac{3}{r_{i j}}+\frac{2}{R}\right)$. Based on Lemma 2, we know $O S_{i j}^{k} \leq \widehat{O F}_{i j}^{k}$ and thus $O F_{i j}^{k} \leq \widehat{O F}_{i j}^{k}+\frac{L_{i j}^{k}}{R}$. By Lemma 5, we have $\widehat{t o B}_{i j}(0, t)-\widehat{t o O}_{i j}(0, t) \leq 2 L$, and by Lemma 3 , we have $t o B_{i j}(0, t)-\widehat{t o B}_{i j}(0, t) \leq$ $L$. Combining them, we obtain $t o B_{i j}(0, t)-\widehat{t o O}_{i j}(0, t) \leq 3 L$. This indicates that, after $P_{i j}^{k}$ arrives at $B_{i j}$, the maximum queue length at $B_{i j}$ in FLAPS-GPS is $3 L$. Because $B_{i j}$ is served by GPS output scheduling with fixed allocated bandwidth $r_{i j}$ in FLAPS-GPS, we have

$$
\widehat{O F}_{i j}^{k} \leq O A_{i j}^{k}+\frac{3 L}{r_{i j}} \leq I F_{i j}^{k}+\frac{3 L}{r_{i j}}
$$

By Lemma 1, IS $S_{i j}^{k} \leq \widehat{I F}_{i j}^{k}$ and thus $I F_{i j}^{k} \leq \widehat{I F}_{i j}^{k}+\frac{L_{i j}^{k}}{R}$. Combing the above equations, we obtain

$$
\begin{aligned}
O F_{i j}^{k} & \leq \widehat{O F}_{i j}^{k}+\frac{L_{i j}^{k}}{R} \\
& \leq I F_{i j}^{k}+\frac{3 L}{r_{i j}}+\frac{L_{i j}^{k}}{R} \\
& \leq \widehat{I F}_{i j}^{k}+\frac{3 L}{r_{i j}}+\frac{2 L_{i j}^{k}}{R} \\
& \leq \widetilde{O F}_{i j}^{k}+L\left(\frac{3}{r_{i j}}+\frac{2}{R}\right)
\end{aligned}
$$

\section{Simulation Results}

We have conducted simulations to verify the analytical results obtained in Section 4 and evaluate the performance of FLAPS.

In the simulations, we consider a $16 \times 16$ buffered crossbar switch without speedup. Each input port and output port has bandwidth of $1 \mathrm{G}$ bps. Since FLAPS can directly handle variable length packets, we set packet length to be uniformly distributed between 40 and 1500 bytes [27]. For bandwidth allocation, we use the same model as that in [15] and [17]. The allocated bandwidth $r_{i j}(t)$ of flow $F_{i j}$ at time $t$ is defined by an unbalanced probability $w$ as follows

$$
r_{i j}(t)= \begin{cases}R\left(w+\frac{1-w}{N}\right), & \text { if } i=j \\ R \frac{1-w}{N}, & \text { if } i \neq j\end{cases}
$$

When $w=0, I n_{i}$ has the same amount of allocated bandwidth at each output port. Otherwise, $I n_{i}$ has more allocated bandwidth at $O u t_{i}$, which is called the hotspot destination. Arrival of a flow $F_{i j}$ is constrained by a leaky bucket $\left(l * r_{i j}(t), \sigma_{i j}\right)$, where $l$ is the effective load. We set the burst size $\sigma_{i j}$ of every flow to a fixed value of 10,000 bytes, and the burst may arrive at any time during a simulation run. We use two traffic patterns in the simulations. For traffic pattern one, each flow has fixed allocated bandwidth during a single simulation run. $l$ is fixed to 1 and $w$ is one of the 11 possible values from 0 to 1 with a step of 0.1 . 
For traffic pattern two, a flow has variable allocated bandwidth. $l$ is one of the 10 possible values from 0.1 to 1 with a step of 0.1 , and for a specific $l$ value, a random permutation of the 11 different $w$ values is used. Each simulation run lasts for 10 seconds.

\subsection{Crosspoint Buffer Size}

Theorem 1 in Section 4.1 gives the bound of the crosspoint buffer size as $4 L$. In this subsection, we look at the maximum and average crosspoint buffer occupancies in the simulations.

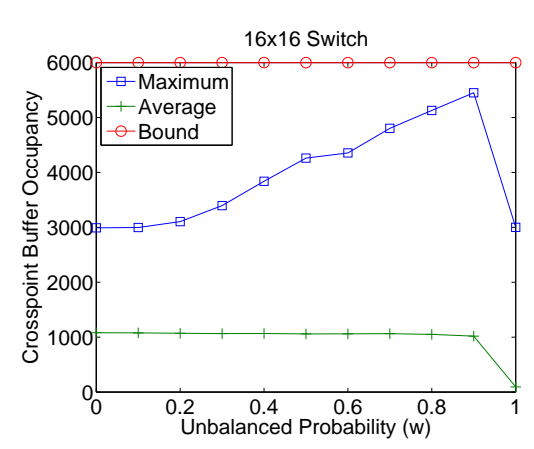

(a)

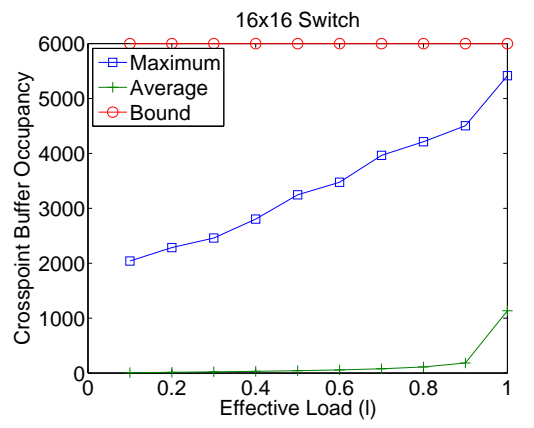

(b)

Fig. 3. Crosspoint buffer occupancy of FLAPS. (a) With different unbalanced probabilities. (b) With different loads.

Figure 3(a) shows the maximum and average crosspoint buffer occupancies under traffic pattern one. As can be see, the maximum occupancy is always smaller than the theoretical bound. It grows as the unbalanced probability increases, but suddenly drops when the unbalanced probability becomes 1 . This is because when the unbalanced probability is 1 , all packets of $I n_{i}$ go to Out $t_{i}$. Thus, there is no switching necessary, and the crosspoint buffer occupancy becomes smaller. For the average occupancy, it does not change significantly with different unbalanced probabilities, and drops when the unbalanced probability becomes 1 for the same reason. We can find that the average occupancy is more affected by the load than the unbalanced probability. Figure 3(b) shows the maximum and average crosspoint buffer occupancies under traffic pattern two. We can see that the maximum occupancy increases as the load increases, but does not exceed the theoretical bound. On the other hand, the average occupancy does not change much and is smaller than 200 bytes before the load increase to 1 . This also confirms the previous observation that the average occupancy is determined by the load.

\subsection{Throughput}

Theorem 2 in Section 4.2 shows that FLAPS achieves strong stability, which implies $100 \%$ throughput. Next, we present the simulation data on throughput of FLAPS. 


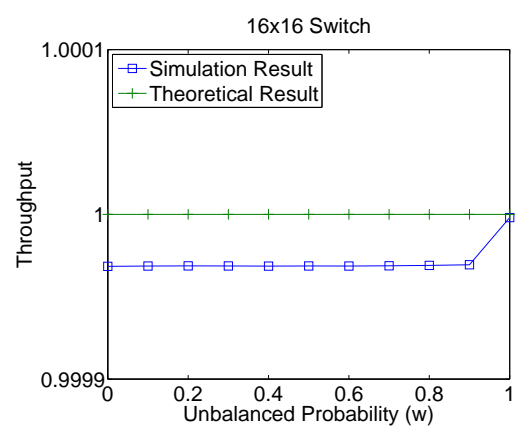

(a)

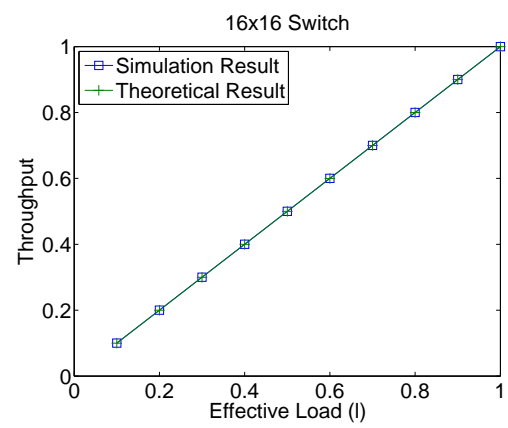

(b)

Fig. 4. Throughput of FLAPS. (a) With different unbalanced probabilities. (b) With different loads.

Figure 4(a) shows the throughput under traffic pattern one. We can see that the throughput for all unbalanced probabilities is greater than 99.99\%, which demonstrates that FLAPS practically achieves 100\% throughput. Figure 4(b) shows the throughput under traffic pattern two. As can be seen, the throughput grows consistently with the effective load, and finally reaches 1 .

\subsection{Jitter}

In this subsection, we present the simulation data on jitter, which is the difference between the packet departure time in FLAPS and GPS. Theorem 3 in Section 4.3 gives the lower bound and upper bound for the jitter of packet $P_{i j}^{k}$. Because Theorem 3 assumes fixed allocated bandwidth $r_{i j}$, we use only traffic pattern one for this part of simulations. Note that the lower bound value depends on the packet length $L_{i j}^{k}$. For easy plotting of the figure, we calculate the jitter lower bound for all packets of flow $F_{i j}$ as follows

$$
-L_{i j}^{k}\left(\frac{1}{r_{i j}}-\frac{2}{R}\right) \geq \begin{cases}-L\left(\frac{1}{r_{i j}}-\frac{2}{R}\right), & \text { if } r_{i j} \leq \frac{R}{2} \\ 0, & \text { if } r_{i j}>\frac{R}{2}\end{cases}
$$

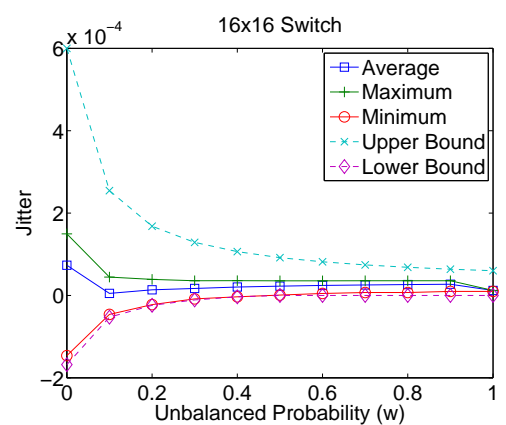

Fig. 5. Jitter of FLAPS with different unbalanced probabilities. 
Figure 5 shows the minimum, maximum, and average jitters of a representative flow $F_{11}$ under traffic pattern one. We can see that the minimum jitter is almost coincident with but always greater than the lower bound, and the maximum jitter is always less than the upper bound. As the unbalanced probability increases, the minimum jitter increases and the maximum jitter decreases. For most of the time, the average jitter is very close to zero, indicating that FLAPS and GPS have similar average packet delay.

\section{Conclusions}

Buffered crossbar switches are special crossbar switches with crosspoint buffers. The introduction of crosspoint buffers greatly simplifies the scheduling process. In this paper, we have proposed the Fair and Localized Asynchronous Packet Scheduling (FLAPS) algorithm, which does not require speedup for the crossbar and can directly handle variable length packets without segmentation and reassembly (SAR). FLAPS uses a time stamp based approach for both input scheduling and output scheduling. We theoretically analyze the performance of FLAPS, and show that it has a crosspoint buffer size bound of $4 L$, independent of the switch size. We also prove that it achieves strong stability, and provides bounded delay guarantees. Finally, we present simulation data and show that they are consistent with the analytical results.

\section{References}

1. J. Kurose and K. Ross, "Computer networking: a top-down approach," Addison Wesley, 4th edition, April 2007.

2. N. McKeown, "A fast switched backplane for a gigabit switched router," Business Communications Review, vol. 27, no. 12, 1997.

3. M. Katevenis and G. Passas, "Variable-size multipacket segments in buffered crossbar (CICQ) architectures," IEEE ICC 2005, Seoul, Korea, May 2005.

4. G. Kornaros, "BCB: a buffered crossBar switch fabric utilizing shared memory," 9th EUROMICRO Conference on Digital System Design, pp. 180-188, Croatia, Aug. 2006.

5. L. Mhamdi, C. Kachris, and S. Vassiliadis, "A reconfigurable hardware based embedded scheduler for buffered crossbar switches," 14th ACM/SIGDA International Symposium on Field Programmable Gate Arrays, pp. 143-149, Monterey, CA, Feb. 2006.

6. I. Papaefstathiou, G. Kornaros, and N. ChrysosUsing, "Buffered crossbars for chip interconnection," 17th Great Lakes Symposium on VLSI, pp. 90-95, Stresa-Lago Maggiore, Italy, Mar. 2007.

7. K. Yoshigoe, K. Christensen, and A. Jacob, "The RR/RR CICQ switch: hardware design for 10-Gbps link speed," 22nd IEEE International Performance, Computing, and Communications Conference, pp. 481-485, Phoenix, AZ, Apr. 2003.

8. S. He et al., "On Guaranteed Smooth Switching for Buffered Crossbar Switches," IEEE/ACM Transactions on Networking vol. 16, no. 3, pp. 718-731, Jun. 2008.

9. B. Magill, C. Rohrs and R. Stevenson, "Output-queued switch emulation by fabrics with limited memory," IEEE Journal on Selected Areas in Communications, vol 21, no. 4, pp. 606-615, May 2003. 
10. L. Mhamdi and M. Hamdi, "Output queued switch emulation by a one-cellinternally buffered crossbar switch," IEEE GLOBECOM 2003, San Francisco, CA, Dec. 2003.

11. D. Stephens and H. Zhang, "Implementing distributed packet fair queueing in a scalable switch architecture," IEEE INFOCOM 1998, San Francisco, CA, March 1998.

12. S. Chuang, S. Iyer, and N. McKeown, "Practical algorithms for performance guarantees in buffered crossbars," IEEE INFOCOM 2005, Miami, FL, Mar. 2005.

13. D. Pan and Y. Yang, "Providing flow based performance guarantees for buffered crossbar switches," IEEE IPDPS 2008, Miami, FL, Apr. 2008.

14. J. Turner, "Strong performance guarantees for asynchronous crossbar schedulers," IEEE/ACM Transactions on Networking, to appear, 2009.

15. R. Rojas-Cessa, E. Oki, Z. Jing, and H. Chao, "CIXB-1: Combined input-oncecell-crosspoint buffered switch," IEEE HPSR 2001, Dallas, TX, Jul. 2001.

16. R. Rojas-Cessa, E. Oki, and H. Chao, "CIXOB-k: Combined input-crosspointoutput buffered packet switch," IEEE Globecom 2001, San Antonio, TX, Nov. 2001.

17. L. Mhamdi and M. Hamdi, "MCBF: a high-performance scheduling algorithm for buffered crossbar switches," IEEE Communications Letters, vol. 7, no. 9, pp. 451453, Sep. 2003

18. X. Zhang and L. Bhuyan, "An efficient scheduling algorithm for combined-inputcrosspoint-queued (CICQ) switches," IEEE Globecom 2004, Dallas, TX, Nov. 2004.

19. M. Katevenis, G. Passas, D. Simos, I. Papaefstathiou, and N. Chrysos, "Variable packet size buffered crossbar (CICQ) switches," Proc. IEEE ICC 2004, Paris, France, June 2004.

20. D. Pan and Y. Yang, "Localized independent packet scheduling for buffered crossbar switches," IEEE Transactions on Computers, vol. 58, no. 2, pp. 260-274, Feb. 2009.

21. A. Parekh and R. Gallager, "A generalized processor sharing approach to flow control in integrated services networks: the single node case," IEEE/ACM Trans. Networking, vol. 1, no. 3, pp. 344-357, Jun. 1993.

22. D. Pan and Y. Yang, "Max-min fair bandwidth allocation algorithms for packet switches," IEEE IPDPS 200\%, Long Beach, CA, Mar. 2007.

23. M. Hosaagrahara and H. Sethu, "Max-min fairness in input-queued switches," IEEE Transactions on Parallel and Distributed Systems, vol. 19, no. 4, pp. 462-475, Apr. 2008.

24. N. McKeown, A. Mekkittikul, V. Anantharam, and J. Walrand, "Achieving 100\% throughput in an input queued switch," IEEE Trans. Commun., vol. 47, no. 8, pp. 1260-1267, Aug. 1999.

25. J. Bennett and H. Zhang, "WF2Q: worst-case fair weighted fair queueing," IEEE INFOCOM 1996, San Francisco, CA, Mar. 1996.

26. E. Leonardi, M. Mellia, F Neri, and M. Marsan, "On the stability of input-queued switches with speed-up," IEEE/ACM Trans. Networking, vol. 9, no. 1, pp. 104-118, Feb. 2001.

27. C. Farleigh et al., "Packet-level traffic measurements from the Sprint IP backbone," IEEE Network, vol. 17, no. 6, pp. 6-16, Nov. 2003. 